\title{
Set-valued risk statistics with the time value of money
}

\section{Fei Sun* • Xiaozhi Fan • Weitao Liu}

\begin{abstract}
The time value of money is a critical factor not only in risk analysis, but also in insurance and financial applications. In this paper, we consider a special class of set-valued risk statistics from the perspective of time value of money. This new risk statistic can be uesd for the quantification of portfolio risk. By further developing the properties related to these risk statistics, we are able to derive representation results for such risk.
\end{abstract}

Keywords risk statistics $\cdot$ set-valued $\cdot$ portfolio $\cdot$ time value

\section{Introduction}

Research on risk is a popular topic in both quantitative and theoretical research, and risk models have attracted considerable attention. The quantitative calculation on risk involves two problems: choosing an appropriate risk model and allocating risk to individual institutions. This has led to further research on risk statistics.

In their seminal paper, Burgert and Rüschendorf (2006) first introduced the concepts of the scalar multivariate coherent and convex risk measures. Chen and $\mathrm{Hu}(2018)$ studied the convex risk measures. However, as pointed out by

\footnotetext{
*Corresponding author

F. Sun

School of Mathematics and Computational Science, Wuyi University, Jiangmen 529020, China

E-mail: fsun.sci@outlook.com

XZ Fan

Changjiang Survey, Planning, Design and Research Co., Ltd, Wuhan 430010, China

E-mail: fanxiaozhi@cjwsjy.com.cn

WT Liu

Guangzhou Institute of Urban Strategy, Guangzhou Academy of Social Sciences, Guangzhou 510410, China

E-mail: cyllwt@aliyun.com
} 
EL Karouii and Ravanelli (2009), the translative invariance axiom may fail once there is any form of uncertainty about interest rates because the money has a time value. For example, when $m$ dollars are added to a future position $X$, the capital requirement at time $t=0$ is reduced by less than $m$ dollars because the value of the money may grow with time. Therefore, it is more appropriate to use cash sub-additivity to replace cash additivity. Therefore, the study of cash sub-additive risk statistics is particularly interesting.

Evaluating risk of a portfolio consisting of several financial positions, Jouini et al. (2004) pointed out that a set-valued risk measure is more appropriate than a scalar risk measure, especially in the case where several different kinds of currencies are involved when one is determining capital requirements for the portfolio. Other studies of set-valued risk measures include those of Ararat et al. (2017), Farkas et al. (2015), Hamel (2009), Hamel and Heyde (2010), Hamel et al. (2011), Hamel et al. (2013), Labuschagne and Offwood-Le Roux (2014), Molchanov and Cascos (2016) and the references therein. A natural set-valued risk statistic can be considered as an empirical (or a data-based) version of a set-valued risk measure.

From the statistical point of view, the behaviour of a random variable can be characterized by its observations, the samples of the random variable. Heyde et al. (2007) and Kou et al. (2013) first introduced the class of natural risk statistics, the corresponding representation results are also derived. An alternative proof of the representation result of the natural risk statistics was also derived by Ahmed et al. (2008). Later, Tian and Suo (2012) obtained representation results for convex risk statistics, and the corresponding results for quasiconvex risk statistics were obtained by Tian and Jiang (2015). However, all of these risk statistics are designed to quantify risk of a single financial position (i.e. a random variable) by its samples. A natural question is determining how to quantify risk of a portfolio by its samples, especially in the situation where different kinds of currencies are possibly involved in the portfolio.

The main focus of this paper is a new class of set-valued risk statistics with the time value of money, named cash sub-additive risk statistics with an axiomatic approach. By further developing the properties related to cash sub-additive risk statistics, we are able to derive representation results for such risk. This new class of risk statistics can be considered as an extension of those introduced by Sun and $\mathrm{Hu}$ (2019) from the empirical (or a data-based) version.

The remainder of this paper is organized as follows. In Sect. 2, we briefly introduce some preliminaries. In Sect. 3. we state the representation result of cash sub-additive risk statistics. In Sect. 4 , we investigate the alternative databased versions of cash sub-additive risk measures. Finally, Sect. 5 discusses the main proof in this paper.

\section{Preliminaries}

In this section, we briefly introduce some preliminaries that are used throughout this paper. Let $d \geq 1$ be a fixed positive integer. The space $\mathbb{R}^{d \times n}$ repre- 
sents the set of financial risk positions. With positive values of $X \in \mathbb{R}^{d \times n}$ we denote the gains while the negative denote the losses. The behavior of the $d$-dimensional random vector $D=\left(X_{1}, \cdots, X_{d}\right)$ in different scenarios is represented by different sets of data observed or generated in those scenarios because specifying accurate models for $D$ is usually very difficult. Here, we suppose that there always exist $l$ scenarios. Let $n_{j}$ be the sample size of $D$ in the $j^{\text {th }}$ scenario, $j=1, \cdots, l$. Let $n:=n_{1}+\cdots+n_{l}$. More precisely, suppose that the behavior of $D$ is represented by a collection of data $X=\left(X_{1}, \cdots, X_{d}\right) \in \mathbb{R}^{n} \times \cdots \times \mathbb{R}^{n}$, where $X_{i}=\left(X^{i, 1}, \cdots, X^{i, l}\right) \in \mathbb{R}^{n}, X^{i, j}=\left(x_{1}^{i, j}, \cdots, x_{n_{j}}^{i, j}\right) \in \mathbb{R}^{n_{j}}$ is the data subset that corresponds to the $j^{\text {th }}$ scenario with respect to $X_{i}$. For each $j=1, \cdots, l, h=1, \cdots, n_{j}, X_{h}^{j}:=\left(x_{h}^{1, j}, x_{h}^{2, j}, \cdots, x_{h}^{d, j}\right)$ is the data subset that corresponds to the $h^{\text {th }}$ observation of $D$ in the $j^{\text {th }}$ scenario, and can be based on historical observations, hypothetical samples simulated according to a model, or a mixture of observations and simulated samples.

In this paper, an element $z$ of $\mathbb{R}^{d}$ is denoted by $z:=\left(z_{1}, \cdots, z_{d}\right)$. An element $X$ of $\mathbb{R}^{d \times n}$ is denoted by $X:=\left(X_{1}, \cdots, X_{d}\right):=\left(x_{1}^{1,1}, \cdots, x_{n_{1}}^{1,1}\right.$, $\left.\cdots, x_{1}^{1, l}, \cdots, x_{n_{l}}^{1, l}, \cdots, x_{1}^{d, 1}, \cdots x_{n_{1}}^{d, 1}, \cdots, x_{1}^{d, l}, \cdots, x_{n_{l}}^{d, l}\right)$. The $d \times n$ dimensional financial positions in $\mathbb{R}^{d \times n}$ have a strong realistic interpretation. This is indeed the case if we consider realistic situations where investors have access to different markets and form multi-asset portfolios in the presence of frictions such as transaction costs, liquidity problems, irreversible transfers, etc.

Let $K$ be a closed convex polyhedral cone of $\mathbb{R}^{d}$ where $K \supseteq \mathbb{R}_{+}^{d}$ where $\mathbb{R}_{+}^{d}:=\left\{\left(x_{1}, \ldots, x_{d}\right) \in \mathbb{R}^{d} ; x_{i}>0,1 \leq i \leq d\right\}$ and $K \cap \mathbb{R}_{-}^{d}=\emptyset$ where $\mathbb{R}_{-}^{d}:=$ $\left\{\left(x_{1}, \ldots, x_{d}\right) \in \mathbb{R}^{d} ; x_{i} \leq 0,1 \leq i \leq d\right\}$. Let $K^{+}$be the positive dual cone of $K$, that is $K^{+}:=\left\{u \in \mathbb{R}^{d}: u^{t r} v \geq 0\right.$ for any $\left.v \in K\right\}$, where $u^{t r}$ means the transpose of $u$. For any $X=\left(X_{1}, \ldots, X_{d}\right), Y=\left(Y_{1}, \ldots, Y_{d}\right) \in \mathbb{R}^{d \times n}, X+Y$ stands for $\left(X_{1}+Y_{1}, \ldots, X_{d}+Y_{d}\right)$ and $a X$ stands for $\left(a X_{1}, \ldots, a X_{d}\right)$ for $a \in \mathbb{R}$. For any $z:=\left(z_{1}, \cdots, z_{d}\right) \in \mathbb{R}^{d}$, denote $K 1_{n}:=\left\{\left(z_{1} 1_{n}, z_{2} 1_{n}, \cdots, z_{d} 1_{n}\right): z \in K\right\}$ and $z 1_{n}:=\{(z, z, \cdots, z): z \in \mathbb{R}\} \in \mathbb{R}^{n}$ where $1_{n}:=(1, \cdots, 1) \in \mathbb{R}^{n}$. By $\left(K 1_{n}\right)^{+}$ we denote the positive dual cone of $K 1_{n}$ in $\mathbb{R}^{d \times n}$, i.e. $\left(K 1_{n}\right)^{+}:=\left\{w \in \mathbb{R}^{d \times n}\right.$ : $w z^{t r} \geq 0$ for any $\left.z \in K\right\}$. The partial order respect to $K$ is defined as $a \leq_{K} b$. Therefore, $b-a \in K$ where $a, b \in \mathbb{R}^{d}$ and $X \leq_{K 1_{n}} Y$ means $Y-X \in K 1_{n}$ where $X, Y \in \mathbb{R}^{d \times n}$.

Let $M:=\mathbb{R}^{m} \times\{0\}^{d-m}$ the linear subspace of $\mathbb{R}^{d}$ for $1 \leq m \leq d$. The introduction of $M$ was considered by Jouini et al. (2004) and Hamel (2009). Denote $M_{+}:=M \cap \mathbb{R}_{+}^{d}$ where $\mathbb{R}_{+}^{d}:=\left\{\left(x_{1}, \ldots, x_{d}\right) \in \mathbb{R}^{d} ; x^{i} \geq 0,1 \leq i \leq d\right\}$. Therefore, a regulator can only accept security deposits in the first $m$ reference instruments. Denote $K_{M}:=K \cap M$ by the closed convex polyhedral cone in $M$, int $K_{M}$ the interior of $K_{M}$ in $M$. We denote $\mathbb{T}_{M}:=\{A \subset M: A=$ $\left.\operatorname{clco}\left(A+K_{M}\right)\right\}$ and $\mathbb{T}_{M^{+}}:=\left\{A \subset K_{M}: A=\operatorname{clco}\left(A+K_{M}\right)\right\}$, where the $\operatorname{clco}(A)$ represents the closed convex hull of $A$. 
By Chen and $\mathrm{Hu}$ (2017), a set-valued convex risk statistic is any map that can be considered as an empirical (or a data-based) version of a setvalued risk measure. The axioms related to this set-valued convex risk statistics $\rho: \mathbb{R}^{d \times n} \rightarrow \mathbb{T}_{M}$ are organized as follows,

A0 Normalized: $K_{M} \subseteq \rho(0)$ and $\rho(0) \cap-i n t K_{M}=\phi$;

A1 Monotonicity: for any $X, Y \in \mathbb{R}^{d \times n}, X-Y \in K 1_{n}$ implies that $\rho(X) \supseteq$ $\rho(Y)$

A2 Translative invariance: for any $X \in \mathbb{R}^{d \times n}$ and $z \in M, \rho\left(X-z 1_{n}\right)=$ $\rho(X)+z$

A3 Convexity: for any $X, Y \in \mathbb{R}^{d \times n}$ and $\lambda \in[0,1], \rho(\lambda(X)+(1-\lambda) Y) \supseteq$ $\lambda \rho(X)+(1-\lambda) \rho(Y)$.

Let $D:=\left(D_{1}, \cdots, D_{d}\right) \in \mathbb{R}^{d \times n}$ be the stochastic discount factor for certain currency $X \in \mathbb{R}^{d \times n}$, i.e. each element of $D$ belones to $[0,1]$.

We end this section with more notations. A function $\rho: \mathbb{R}^{d \times n} \rightarrow \mathbb{T}_{M}$ is called proper if $\operatorname{dom} \rho:=\left\{X \in \mathbb{R}^{d \times n}: \rho(X) \neq \emptyset\right\} \neq \emptyset$ and $\rho(X) \neq M$ for all $X \in \operatorname{dom} \rho . \rho$ is said to be $\operatorname{closed}$ if $\operatorname{graph} \rho$ is a closed set with respect to the product topology in $\mathbb{R}^{d \times n} \times M$.

\section{Empirical versions of set-valued cash sub-additive risk measures}

Since the time value of money is a critical factor in risk analysis, we will consider a special class of set-valued risk statistics, named cash sub-additive risk statistics. Note that these set-valued risk statistics are the empirical versions of set-valued cash sub-additive risk measures introduced by Sun and $\mathrm{Hu}$ (2019).

In this section, we state the representation results of cash sub-additive risk statistics. However, our viewpoint is not the same as Chen and $\mathrm{Hu}$ (2017). Instead, we start from the relation between set-valued convex risk statistics and set-valued cash sub-additive risk statistics.

We begin with the axioms related to cash sub-additive risk statistics.

Definition 31 A set-valued cash sub-additive risk statistic is a set-valued function $R: \mathbb{R}^{d \times n} \rightarrow \mathbb{T}_{M}$ that satisfies $\mathbf{A 0}, \mathbf{A} \mathbf{1}, \mathbf{A} \mathbf{3}$ and the following property.

A4 Cash sub-additivity: for any $X \in \mathbb{R}^{d \times n}$ and $z \in K_{M}$,

$$
R\left(X+z 1_{n}\right) \subseteq R(X)-z \quad \text { or } \quad R\left(X-z 1_{n}\right) \supseteq R(X)+z .
$$

In fact, the cash sub-additivity of set-valued cash sub-additive risk statistic is derived from the idea of the time value of money. In this context, we define a set-valued risk statistic in the discounted position. 
Definition 32 Let $D \in \mathbb{R}^{d \times n}$ be the discount factor. A set-valued discount risk statistic, say $\bar{R}$, is a set-valued convex risk statistic which is defined in the discounted factor $D X$ where $X \in \mathbb{R}^{d \times n}$

By set-valued discount risk statistic $\bar{R}$, we can define a set-valued convex function by $R(X):=\bar{R}(D X)$. As $\bar{R}$ is a set-valued convex risk statistic, for any $z \in K_{M}$, we have

$$
R\left(X+z 1_{n}\right)=\bar{R}\left(D X+D z 1_{n}\right) \subseteq \bar{R}\left(D X+z 1_{n}\right)=\bar{R}(D X)-z=R(X)-z .
$$

This property of $R$ is due to the time value of money. That is to say that $R$ is expressed in terms of the current numéraire but defined in the future financial positions with the future numéraire.

We now introduce a special case of set-valued cash sub-additive risk statistics that is said to be set-valued convex loss-based risk statistics, see Sun et al. (2018). Note that the scalar case of convex loss-based risk measures was studied by Chen et al.(2018) and Cont et al. (2013). The following definition is derived from Sun et al. (2018).

Definition 33 A set-valued loss-based risk statistic is a mapping $\varrho: \mathbb{R}^{d \times n} \rightarrow$ $\mathbb{T}_{M^{+}}$that satisfies the following properties.

B0 Normalization: $K_{M} \subseteq \varrho(0)$ and $\varrho(0) \cap-$ int $K_{M}=\phi$;

$B 1$ Cash losses: for any $z \in K_{M}, z \in \varrho\left(-z 1_{n}\right)$;

B2 Monotonicity: for any $X, Y \in \mathbb{R}^{d \times n}, X-Y \in K 1_{n}$ implies $\varrho(X) \supseteq \varrho(Y)$;

B3 Loss-dependence: for any $\left.X \in \mathbb{R}^{d \times n}, \varrho(X)=\varrho\left(X \wedge_{K 1_{n}}\right)\right)$, where

$$
X \wedge_{K 1_{n}} 0:=\left\{\begin{array}{l}
X, X \notin K 1_{n}, \\
0, X \in K 1_{n} .
\end{array}\right.
$$

B4 Convexity: for any $\lambda \in[0,1]$ and $X, Y \in \mathbb{R}^{d \times n}, \varrho(\lambda X+(1-\lambda) Y) \supseteq$ $\lambda \varrho(X)+(1-\lambda) \varrho(Y)$.

Remark 31 The set-valued loss-based risk statistics start from the point of regulators. Namely, the regulators almost only focus on the loss of investment rather than revenue. Especially, the axiom of translation invariance in coherent and convex risk statistics will definitely fail when we only to deal with the lossbased risk. Therefore, the loss-based risk is particularly interesting.

We claim that the set-valued loss-based risk statistics are the special cases of set-valued cash sub-additive risk statistics. Indeed, for any $X \in \mathbb{R}^{d \times n}$, $z \in K_{M}$ and $\varepsilon \in(0,1)$, we have

$$
\begin{aligned}
\varrho\left((1-\varepsilon) X-z 1_{n}\right) & =\varrho\left((1-\varepsilon) X+\varepsilon\left(-\frac{z}{\varepsilon}\right) 1_{n}\right) \\
& \supseteq(1-\varepsilon) \varrho(X)+\varepsilon \varrho\left(-\frac{z}{\varepsilon} 1_{n}\right) \\
& \supseteq(1-\varepsilon) \varrho(X)+z,
\end{aligned}
$$


where the last inclusion is due to the property of cash losses. Therefore, by the arbitrariness of $\varepsilon$, we conclude that

$$
\varrho\left(X-z 1_{n}\right) \supseteq \varrho(X)+z .
$$

This indicates that $\varrho$ satisfies the property A4. Therefore, $\varrho$ is cash subadditive. Next, we introduce an example of set-valued loss-based risk statistic called $A V @ R^{\text {loss }}$.

Example 31 (Loss-based average value at risk) For any $X \in \mathbb{R}^{d \times n}$ and $0<$ $\alpha<1$, we define $A V @ R_{\alpha}^{\text {loss }}$ as

$$
A V @ R_{\alpha}^{\text {loss }}(X)=\inf _{z \in \mathbb{R}^{d}}\left\{\frac{1}{\alpha}\left(-\left.\left(X \wedge_{K 1_{n}} 0\right)\right|_{M}+z\right)^{+}-z\right\}+\mathbb{R}_{+}^{m} .
$$

It is clear that $A V @ R^{\text {loss }}$ satisfies all the properties of Definition 33. So $A V @ R^{\text {loss }}$ is a set-valued loss-based risk statistic, and hence it is also cash sub-additive.

We now need to derive the representation results of cash sub-additive risk statistics. Any pair $(X, \mu)$, where $X \in \mathbb{R}^{d \times n}$ and $\mu \in M$, can be viewed as the coordinates of a $\bar{X} \in \mathbb{R}^{d \times n}$ in $\mathcal{T}:=\{0,1\}$ with the element $\theta$,

$$
\bar{X}(\theta):=X I_{\{1\}}(\theta)+\mu 1_{n} I_{\{0\}}(\theta) .
$$

For any $\bar{X}, \bar{Y} \in \mathcal{X}$ with $\bar{X}=X I_{\{1\}}+\mu_{1} 1_{n} I_{\{0\}}$ and $\bar{Y}=Y I_{\{1\}}+\mu_{2} 1_{n} I_{\{0\}}$, where $X, Y \in \mathbb{R}^{d \times n}, \mu_{1}, \mu_{2} \in M$, we define the order as $\bar{X}-\bar{Y} \in K 1_{n}$ in the case of $Y \leq_{K 1_{n}} X$ and $\mu_{2} \leq_{K} \mu_{1}$.

We begin with stating the relation between a set-valued convex risk statistic and a set-valued cash sub-additive risk statistic.

Proposition 31 Given a set-valued cash sub-additive risk statistic $R$ in $\mathbb{R}^{d \times n}$ with $0 \in R(0)$, we define a set-valued risk statistic $\rho$ as follows. For any $\bar{X}$ defined as (3.1), with $X \in \mathbb{R}^{d \times n}, \mu \in M$,

$$
\rho(\bar{X}):=R\left(X-\mu 1_{n}\right)-\mu .
$$

Then $\rho$ is a set-valued convex risk statistic with $\rho(0)=0$ and $\rho\left(X I_{\{1\}}\right)=R(X)$.

Before we state the representation results of cash sub-additive risk statistics, the representation results of set-valued convex risk statistics should be recalled. The set-valued convex risk statistics were studied by $\mathrm{Chen}$ and $\mathrm{Hu}$ (2017). We now only state their main results and omit their proofs.

Proposition $32 \rho: \mathbb{R}^{d \times n} \rightarrow \mathbb{T}_{M}$ is a set-valued proper closed convex risk statistic in the case of there is a function $-\alpha:\left(K 1_{n}\right)^{+} \cap \mathbb{R}_{+}^{d \times n} \rightarrow \mathbb{T}_{M}$ such that for any $\bar{X} \in \mathbb{R}^{d \times n}$,

$$
\rho(\bar{X})=\bigcap_{v \in\left(K 1_{n}\right)^{+} \cap \mathbb{R}_{+}^{d \times n}}\left\{-\alpha(v)+S_{v}(-\bar{X})\right\},
$$


where

$$
S_{v}(-\bar{X}):=\left\{u \in M: v^{\operatorname{tr}}\left(\bar{X}+u 1_{n}\right) \geq 0\right\} .
$$

In particular, 3.3 ) is satisfied with $-\alpha$ replaced by $-\alpha_{\min }$ with

$$
-\alpha_{\min }(v):=c l \bigcup_{\bar{Z} \in\left\{\bar{X} \in \mathbb{R}^{d \times n}: 0 \in \rho(\bar{X})\right\}} S_{v}(\bar{Z}) .
$$

Using Propositions 32, we are able to derive the representation results of set-valued cash sub-additive risk statistics.

Theorem 31 Any set-valued proper closed cash sub-additive risk statistic $R$ in $\mathbb{R}^{d \times n}$ has the following form. For any $X \in \mathbb{R}^{d \times n}$,

$$
R(X)=\bigcap_{v \in\left(K 1_{n}\right)^{+} \cap \mathbb{R}_{+}^{d \times n}}\left\{-\gamma(v)+\mathfrak{S}_{v}(-X)\right\},
$$

where

$$
-\gamma:\left(K 1_{n}\right)^{+} \cap \mathbb{R}_{+}^{d \times n} \rightarrow \mathbb{T}_{M}
$$

and

$$
\mathfrak{S}_{v}(-X):=\left\{u \in M: v^{\operatorname{tr}}\left(X I_{\{1\}}+u 1_{n}\right) \geq 0\right\} .
$$

In particular, (3.4) is satisfied with $-\gamma$ replaced by $-\gamma_{\min }$ with

$$
-\gamma_{\min }(v):=c l \underset{Z \in\left\{X \in \mathbb{R}^{d \times n}: 0 \in R(X)\right\}}{\mathfrak{S}_{v}(Z) .}
$$

\section{Alternative data-based versions of set-valued cash sub-additive risk measures}

In this section, we develop another framework of data-based versions of cash sub-additive risk measures. This framework is a little different from the previous one. However, almost all the arguments are the same as those in the previous section. Therefore, we only state the corresponding notations and results, and omit all the proofs and relevant explanations.

We replace $M$ by $\widetilde{M} \in \mathbb{R}^{d \times n}$ that is a linear subspace of $\mathbb{R}^{d \times n}$. We also replace $K$ by $\widetilde{K} \in \mathbb{R}^{d \times n}$ that is a closed convex polyhedral cone where $\widetilde{K} \supseteq \mathbb{R}_{+}^{d \times n}$. The partial order respect to $\widetilde{K}$ is defined as $X \leq_{\widetilde{K}} Y$, which means $Y-X \in \widetilde{K}$. Denote $\widetilde{K}_{\widetilde{M}}:=\widetilde{K} \cap \widetilde{M}$ by the closed convex polyhedral cone in $\widetilde{M}$, int $\widetilde{K}_{\widetilde{M}}$ the interior of $\widetilde{K}_{\widetilde{M}}$ in $\widetilde{M}$. We denote $\mathbb{T}_{\widetilde{M}}:=\{\widetilde{A} \subset \widetilde{M}: \widetilde{A}=$ $\left.\operatorname{clco}\left(\widetilde{A}+\widetilde{K}_{\widetilde{M}}\right)\right\}$.

We begin with recalling the axioms related to data-based versions of setvalued convex risk measures in Chen and $\mathrm{Hu}$ (2017). 
Definition 41 A set-valued data-based convex risk statistic is a function $\widetilde{\rho}$ : $\mathbb{R}^{d \times n} \rightarrow \mathbb{T}_{\widetilde{M}}$ that satisfies the following properties,

C0 Normalization: $\widetilde{K}_{\widetilde{M}} \subseteq \widetilde{\rho}(0)$ and $\widetilde{\rho}(0) \cap-i n t \widetilde{K}_{\widetilde{M}}=\phi$;

C1 Monotonicity: for any $X_{1}, X_{2} \in \mathbb{R}^{d \times n}, X_{1}-X_{2} \in \mathbb{R}^{d \times n} \cap \widetilde{K}$ implies that $\widetilde{\rho}\left(X_{1}\right) \supseteq \widetilde{\rho}\left(X_{2}\right)$;

C2 Translative invariance: for any $X \in \mathbb{R}^{d \times n}$ and $z \in \widetilde{M}, \widetilde{\rho}(X-z)=\widetilde{\rho}(X)+z$;

C3 Convexity: for any $X, Y \in \mathbb{R}^{d \times n}, \lambda \in[0,1], \widetilde{\rho}(\lambda(X)+(1-\lambda) Y) \supseteq \lambda \widetilde{\rho}(X)+$ $(1-\lambda) \widetilde{\rho}(Y)$.

We now define the set-valued data-based cash sub-additive risk statistics.

Definition 42 A set-valued data-based cash sub-additive risk statistic is a function $\widetilde{R}: \mathbb{R}^{d \times n} \rightarrow \mathbb{T}_{\widetilde{M}}$ that satisfies C0, C1, C3 and the following property. C4 Cash sub-additivity: for any $X \in \mathbb{R}^{d \times n}$ and $z \in \widetilde{K}_{\widetilde{M}}$,

$$
\widetilde{R}(X+z) \subseteq \widetilde{R}(X)-z \quad \text { or } \quad \widetilde{R}(X-z) \supseteq \widetilde{R}(X)+z .
$$

We need more notions. Any pair $(X, \widetilde{\mu})$, where $X \in \mathbb{R}^{d \times n}$ and $\widetilde{\mu} \in \widetilde{M}$, can be viewed as the coordinates of a $\widetilde{X} \in \mathbb{R}^{d \times n}$ in $\mathcal{T}:=\{0,1\}$ with the element $\theta$,

$$
\widetilde{X}(\theta):=X I_{\{1\}}(\theta)+\widetilde{\mu} I_{\{0\}}(\theta) .
$$

Proposition 41 Given a set-valued data-based cash sub-additive risk statistic $\widetilde{R}$ in $\mathbb{R}^{d \times n}$ with $0 \in \widetilde{R}(0)$, we define a set-valued data-based risk statistic $\widetilde{\rho}$ as follows. For any $\widetilde{X}$ defined as (4.1) where $X \in \mathbb{R}^{d \times n}, \widetilde{\mu} \in \widetilde{M}$,

$$
\widetilde{\rho}(\widetilde{X}):=\widetilde{R}(X-\widetilde{\mu})-\widetilde{\mu} .
$$

Then $\widetilde{\rho}$ is a set-valued data-based convex risk statistic with $\widetilde{\rho}(0)=0$ and $\widetilde{\rho}\left(X I_{\{1\}}\right)=\widetilde{R}(X)$.

The representation results of set-valued data-based convex risk statistics were studied in Chen and $\mathrm{Hu}$ (2017). We now state the main results of this section.

Theorem 41 Any set-valued data-based proper closed cash sub-additive risk statistic $\widetilde{R}$ in $\mathbb{R}^{d \times n}$ has the following form. For any $X \in \mathbb{R}^{d \times n}$,

$$
\widetilde{R}(X)=\bigcap_{\widetilde{v} \in \widetilde{K}^{+} \cap \mathbb{R}_{+}^{d \times n}}\left\{-\widetilde{\gamma}(\widetilde{v})+\widetilde{\mathfrak{S}}_{\widetilde{v}}(-X)\right\},
$$

where

$$
-\widetilde{\gamma}: \widetilde{K}^{+} \cap \mathbb{R}_{+}^{d \times n} \rightarrow \mathbb{T}_{\widetilde{M}}
$$

and

$$
\widetilde{\mathfrak{S}}_{\widetilde{v}}(-X):=\left\{\widetilde{u} \in \widetilde{M}: \widetilde{v}^{\operatorname{tr}}\left(X I_{\{1\}}+\widetilde{u}\right) \geq 0\right\} .
$$

In particular, (4.3) is satisfied with $-\widetilde{\gamma}$ replaced by $-\widetilde{\gamma}_{\min }$ with

$$
-\widetilde{\gamma}_{\min }(\widetilde{v}):=c l \bigcup_{Z \in\left\{X \in \mathbb{R}^{d \times n}: 0 \in \widetilde{R}(X)\right\}} \mathfrak{S}_{\widetilde{v}}(Z) .
$$




\section{Proofs of main results}

In this section, we provide all the proofs of the results stated in Sect. 3 .

Proof of Proposition 31. It is easy to check that $\rho(0)=0, \rho\left(X I_{\{1\}}\right)=R(X)$ and $\rho$ satisfies the property of A0. Next, we derive that $\rho$ satisfies properties of A1, A2 and A3.

A1. Monotonicity: for any $\bar{X}=X I_{\{1\}}+\mu_{1} 1_{n} I_{\{0\}}$ and $\bar{Y}=Y I_{\{1\}}+\mu_{2} 1_{n} I_{\{0\}}$, where $X, Y \in \mathbb{R}^{d \times n}, \mu_{1}, \mu_{2} \in M$ with $\bar{X}-\bar{Y} \in K 1_{n}$, then

$\rho(\bar{X})=R\left(X-\mu_{1} 1_{n}\right)-\mu_{1} \supseteq R\left(X-\mu_{2} 1_{n}\right)-\mu_{2} \supseteq R\left(Y-\mu_{2} 1_{n}\right)-\mu_{2}=\bar{\rho}(\bar{Y})$,

which shows that $\rho$ is monotone.

A2. Translative invariance: for any $b \in M, \bar{X}=X I_{\{1\}}+\mu 1_{n} I_{\{0\}}$ where $X \in$ $\mathbb{R}^{d \times n}$ and $\mu \in M$,

$$
\begin{aligned}
\rho\left(\bar{X}+b 1_{n}\right) & =\rho\left(\left(X+b 1_{n}\right) I_{\{1\}}+\left(\mu 1_{n}+b 1_{n}\right) I_{\{0\}}\right) \\
& =R\left(X+b 1_{n}-(\mu+b) 1_{n}\right)-\mu-b \\
& =R\left(X-\mu 1_{n}\right)-\mu-b \\
& =\rho(\bar{X})-b,
\end{aligned}
$$

which shows that $\rho$ satisfies the translative invariance.

A3. Convexity: for any $\lambda \in(0,1), \bar{X}=X I_{\{1\}}+\mu_{1} 1_{n} I_{\{0\}}$ and $\bar{Y}=Y I_{\{1\}}+$ $\mu_{2} 1_{n} I_{\{0\}}$, where $X, Y \in \mathbb{R}^{d \times n}, \mu_{1}, \mu_{2} \in M$,

$$
\begin{aligned}
& \rho(\lambda \bar{X}+(1-\lambda) \bar{Y}) \\
= & \rho\left((\lambda X+(1-\lambda) Y) I_{\{1\}}+\left(\lambda \mu_{1}+(1-\lambda) \mu_{2}\right) 1_{n} I_{\{0\}}\right) \\
= & R\left((\lambda X+(1-\lambda) Y)-\left(\lambda \mu_{1}+(1-\lambda) \mu_{2}\right) 1_{n}\right)-\lambda \mu_{1}-(1-\lambda) \mu_{2} \\
= & R\left(\lambda\left(X-\mu_{1} 1_{n}\right)+(1-\lambda)\left(Y-\mu_{2} 1_{n}\right)\right)-\lambda \mu_{1}-(1-\lambda) \mu_{2} \\
\supseteq & \lambda R\left(X-\mu_{1} 1_{n}\right)+(1-\lambda) R\left(Y-\mu_{2} 1_{n}\right)-\lambda \mu_{1}-(1-\lambda) \mu_{2} \\
= & \lambda \rho(\bar{X})+(1-\lambda) \rho(\bar{Y}),
\end{aligned}
$$

which shows that $\rho$ is convex.

Proof of Proposition 32. See Chen and Hu (2017).

Proof of Proposition 31. From Proposition 31, we can define a set-valued convex risk statistic $\rho$ in $\mathbb{R}^{d \times n}$ by $R$, such that $\rho\left(X I_{\{1\}}\right)=R(X)$ for any $X \in \mathbb{R}^{d \times n}$. Indeed, as $0 \in \mathbb{R}^{d}$, we have $X I_{\{1\}} \in \mathbb{R}^{d \times n}$. Therefore, from Proposition 32 .

$$
R(X)=\rho\left(X I_{\{1\}}\right)=\bigcap_{v \in\left(K 1_{n}\right)^{+} \cap \mathbb{R}_{+}^{d \times n}}\left\{-\alpha(v)+S_{v}\left(-X I_{\{1\}}\right)\right\},
$$


where $-\alpha:\left(K 1_{n}\right)^{+} \cap \mathbb{R}_{+}^{d \times n} \rightarrow \mathbb{T}_{M}$ and

$$
S_{v}(-\bar{X}):=\left\{u \in M: v^{t r}\left(\bar{X}+u 1_{n}\right) \geq 0\right\} .
$$

In particular, the function $-\alpha$ can be replaced by $-\alpha_{\min }$ where

$$
-\alpha_{\min }(v):=c l \bigcup_{Z \in\left\{X \in \mathbb{R}^{d \times n}: 0 \in \rho\left(X I_{\{1\}}\right)\right\}} S_{v}\left(Z I_{\{1\}}\right) .
$$

We now denote

$$
\mathfrak{S}_{v}(X):=\left\{u \in M: v^{\operatorname{tr}}\left(-X I_{\{1\}}+u 1_{n}\right) \geq 0\right\}
$$

Therefore, the set-valued cash sub-additive risk statistic $R$ can be expressed as

$$
R(X)=\bigcap_{v \in\left(K 1_{n}\right)^{+} \cap \mathbb{R}_{+}^{d \times n}}\left\{-\gamma(v)+\mathfrak{S}_{v}(-X)\right\},
$$

where $-\gamma:\left(K 1_{n}\right)^{+} \cap \mathbb{R}_{+}^{d \times n} \rightarrow \mathbb{T}_{M}$ and can replaced by $-\gamma_{\text {min }}$ where

$$
-\gamma_{\min }(v):=c l \underset{Z \in\left\{X \in \mathbb{R}^{d \times n}: 0 \in R(X)\right\}}{\bigcup_{v}(Z) .}
$$

\section{Ethical Approval}

This article does not contain any studies with human participants or animals performed by any of the authors.

\section{Funding Details}

This work is supported by Guangdong Basic and Applied Basic Research Foundation (2020A1515110671), Jiangmen Basic and Applied Basic Research Foundation (2021030100070004859) and Young Innovative Talents Project of Guangdong Province (2019KQNCX156).

\section{Conflict of Interest}

The authors declare that the research was conducted in the absence of any commercial or financial relationships that could be construed as a potential conflict of interest.

This manuscript has been released as a pre-print at arXiv: 1905.00486. 


\section{Informed Consent}

Informed consent was obtained from all individual participants included in the study.

\section{Author Contributions}

Conceptualization, F.Sun; Formal analysis, F.Sun and XZ.Fan; Writingoriginal draft preparation, F.Sun and WT.Liu; Writing-review and editing, F.Sun and XZ.Fan; Funding acquisition, F.Sun.

\section{References}

1. Ahmed S, Filipović D, Svindland G (2008) A note on natural risk statistics, Oper. Res. Lett. 36, 662-664.

2. Ararat C, Hamel AH, Rudloff B (2017) Set-valued shortfall and divergence risk measures, arXiv: 1405.4905v2 [q-fin.RM] 19 May.

3. Burgert C, Rüschendorf L (2006) Consistent risk measures for portfolio vectors, Insurance Math. Econom. 38, 289-297.

4. Chen Y, Hu Y (2017) Set-valued risk statistics with scenario analysis, Statist.Probab.Lett, 131, 25-37.

5. Chen Y, Sun F, Hu Y (2018) Coherent and convex loss-based risk measures for portfolio vectors, Positivity, 22(1), 399-414.

6. Chen Z, Hu Q (2018) On coherent risk measures induced by convex risk measures, Methodol Comput Appl Probab, 20, 673-698.

7. Cont R, Deguest R, He XD (2013) Loss-based risk measures, Stat. Risk Model., 30(2), 133-167.

8. EL Karouii N, Ravanelli C (2009) Cash subadditive risk measures and Interest rate ambiguity, Math. Finance, 19, 561-590.

9. Farkas W, Koch-Medina P, Munari C (2015) Measuring risk with multiple eligible assets, Math. Financ. Econ. 9(1), 3-27.

10. Hamel AH (2009) A duality theory for set-valued functions I: Fenchel conjugation theory, Set-Valued Var. Anal. 17(2), 153-182.

11. Hamel AH, Heyde F (2010) Duality for set-valued measures of risk, SIAM J. Financial Math. 1(1), 66-95.

12. Hamel AH, Heyde F, Rudloff B (2011) Set-valued risk measures for conical market models, Math. Financ. Econ. 5(1), 1-28.

13. Hamel AH, Rudloff B, Yankova M (2013) Set-valued average value at risk and its computation, Math. Financ. Econ. 7(2), 229-246.

14. Heyde CC, Kou SG, Peng XH (2007) What is a good external risk measure: Bridging the gaps between robustness, subadditivity, and insurance risk measures, Working paper, Columbia University.

15. Jouini E, Meddeb M, Touzi N (2004) Vector-valued coherent risk measures, Finance Stoch. 8(4), 531-552.

16. Kou SG, Peng XH, Heyde CC (2013) External risk measures and basel accords, Math. Oper. Res. 38, 393-417

17. Labuschagne CCA, Offwood-Le Roux TM (2014) Representations of set-valued risk measures definded on the $l$-tensor product of Banach lattices, Positivity, 18(3), 619-639.

18. Molchanov I, Cascos I (2016) Multivariate risk measures: a constructive approach based on selections, Math. Finance, 26(4), 867-900.

19. Sun F, Chen Y, Hu Y (2018) Set-valued loss-based risk measures, Positivity, 22(3), 859-871. 
20. Sun F, Hu Y (2019) Set-valued cash sub-additive risk measures, Probab. Engrg. Inform Sci. 33(2), 241-257.

21. Tian D, Jiang L (2015) Quasiconvex risk statistics with scenario analysis, Math. Financ. Econ. 9, 111-121.

22. Tian D, Suo X (2012) A note on convex risk statisitc, Oper. Res. Lett. 40, 551-553. 